\title{
Channel gating pore: a new therapeutic target
}

\author{
Cell Research (2013) 23:1067-1068. doi:10.1038/cr.2013.89; published online 9 July 2013
}

Each subunit of voltage-gated cation channels comprises a voltagesensing domain and a pore region. In a paper recently published in Cell Research, Li et al. showed that the gating charge pathway of the voltage sensor of the $\mathrm{KCNQ} 2 \mathrm{~K}^{+}$channel can accommodate small opener molecules and offer a new target to treat hyperexcitability disorders.

Voltage-gated cation channels (VGCCs) are key players of many vital functions and their genetic defects in humans can lead to severe diseases, called "channelopathies" [1]. Each channel $\alpha$ subunit possesses two main transmembrane modules, a voltage-sensing domain (VSD) and a pore region. VSDs are membrane protein modules comprising four membrane-spanning segments (S1-S4) endowed with charged amino acids, also called gating charges [2]. Although the precise nature and extent of the conformational rearrangement of the VSD is still debated, it is commonly recognized that four highly conserved arginine residues along S4 (R1, R2, $\mathrm{R} 3$, and R4) mainly contribute to the voltage-driven gating charge transfer during channel activation [3]. The gating charges reside in aqueous crevices, and they translocate across a focused electric field spanned by a short distance where hydrophobic residues form a hydrophobic plug occluding a "gating pore". Along this narrow hydrophobic region, the positive charges in $\mathrm{S} 4$ are stabilized by electrostatic interactions with negative countercharges in segments S2 and S3, water in the crevices and negatively charged phospholipids [3]. In voltage-gated $\mathrm{K}^{+}$channels, a highly conserved phenylalanine residue located at the bottom of the S2 segment faces the intracellular side of the hydrophobic plug [4]. This aromatic residue forms the extracellular lid of an occluded site that separates the extracellular and intracellular water-filled crevices of the VSD and forms the charge-transfer center that catalyzes movement of the gating charges [5].

Molecules that target ion channel proteins have been very instrumental in adding drugs to the medicinal therapeutic arsenal as well as in providing tools to dissect the mechanisms of ion channel gating. However, so far, the pharmacological toolbox has focused only on the pore and gate regions of ion channels, both from a fundamental biophysical perspective and from a therapeutic outlook. In contrast, the VSD was virtually not targeted with small ligand molecules neither for therapeutic purposes nor for deciphering ion channel gating, though it is the target of various toxins. A recent study in Cell Research by Li et al. [6] showed that the gating charge pathway or "gating pore" of the therapeutically relevant voltagedependent $\mathrm{K}^{+}$channel KCNQ2 could accommodate small opener molecules, thereby offering a new target to treat hyperexcitability disorders.

Using a comprehensive approach employing homology modeling, molecular docking, molecular dynamics (MD) simulation, mutagenesis and electrophysiology, Li et al. [6] identified an activator-binding pocket in the occluded gating pore of KCNQ2. First, a small opener molecule ztz240 recently discovered by the same group was used as a probe to determine by scanning mutagenesis the binding model of ligands in the KCNQ2 gating charge pathway (Figure 1). Among the mutational hits, several VSD mutants in S2 and S4 dramatically decreased the opener activity of ztz240, including the mutant of the conserved phenylalanine (F137A) in S2 forming the hydrophobic plug of the KCNQ2 gating pore. Exploiting the mutational constraints and using a flexible docking program, $\mathrm{Li}$ and coworkers built a docking model for the opener ztz240 onto a structural homology model of KCNQ2 that was based on the open state structure of Kv1.2 channel. They could precisely determine the orientation of the ligand into the binding pocket by wisely synthesizing two chemical derivatives of ztz240 and testing them on KCNQ2 channel activity. Next, they further optimized the docking model by MD simulation of the ligand-channel complex embedded into phospholipids. The docking model defined a broad pocket, spreading from the extracellular entrance of the VSD groove to the bottom of the gating pore with the ligand engaged in a wide array of hydrophobic, H-bonding and electrostatic interactions. Adopting a very elegant strategy, Li et al. set out to screen a structure-based virtual library of about 200000 chemicals that were selected to fit the newly identified ligand-binding pocket by a docking approach. The purpose was to discover new KCNQ2 channel openers and eventually provide lead optimization (Figure 1). Out of 25 hits selected by bioassays, nine compounds showed significant $\mathrm{KCNQ} 2$ opener activity with $\mathrm{EC}_{50}$ in the micromolar range. Remarkably, as an ultimate validation, these newly discovered KCNQ2 channel openers 


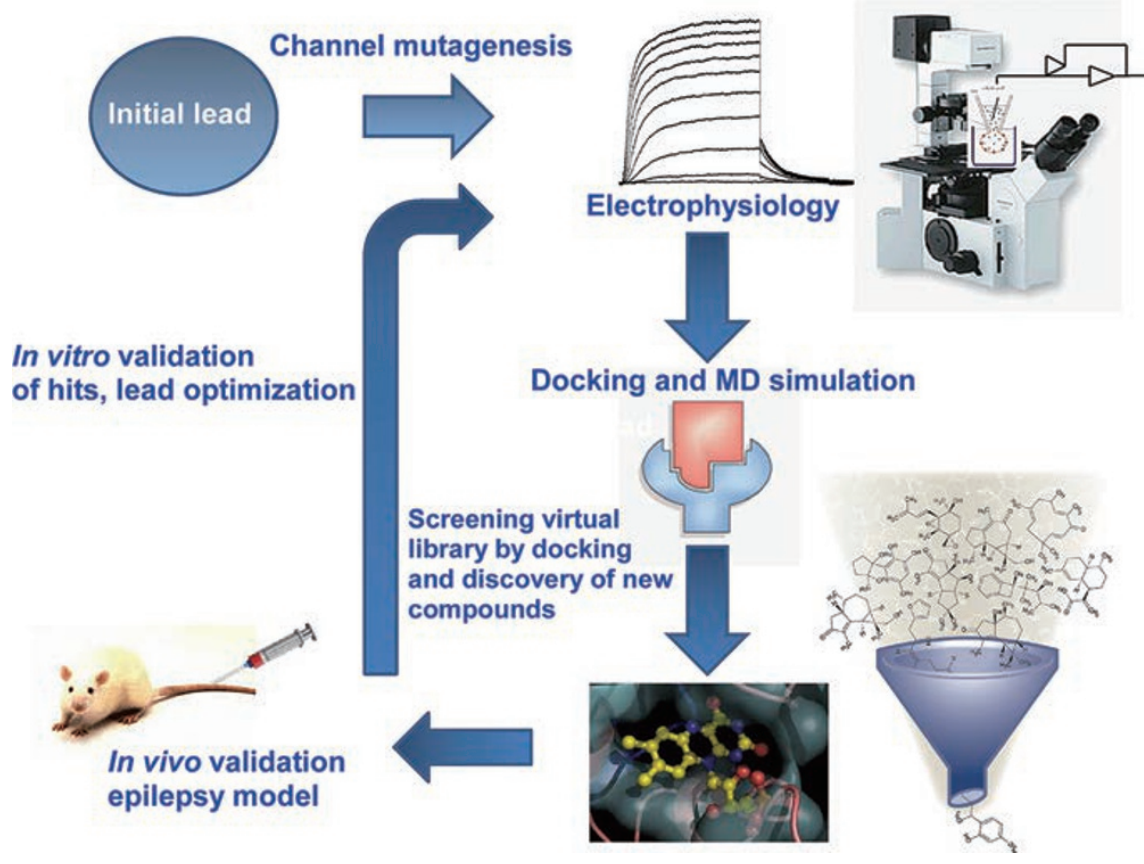

Figure 1 Cartoon summarizing the strategy used to discover new channel opener molecules. Following synthesis of an initial lead compound, a scanning channel mutagenesis and subsequent electrophysiological testing of the lead are performed on the mutants. This step allows identification of crucial residues for lead activity. Next, flexible docking and MD simulations are carried out to define the ligand-binding pocket. Then, a screen of a structure-based virtual library is performed where chemicals are selected to fit the newly identified ligand-binding pocket by a docking approach. Following this stage, the hits are validated in vitro by electrophysiology, which allows discovery of new compounds and lead optimization. The novel active compounds are tested for validation in vivo using animal models. This strategy could be applied to the discovery of any modulator in any kind of ion channel.

demonstrated an excellent anti-epileptic activity in two different murine models of epilepsy.

The study of Li et al. identifies a new therapeutic target, a ligand-binding site in the gating pore of KCNQ2 channels at the heart of the gating machinery where the electric field is highly focused. The opener-binding pocket with a volume of about $170 \AA^{3}$ extends deeply inside the VSD and is different from the site of another compound $\mathrm{NH} 29$, previously reported to locate in a more superficial

region of the VSD [7]. The clever approach of $\mathrm{Li}$ and co-workers provides a $36 \%$ hit rate of virtual screening, which is much higher than hit rates of cell-based high throughput screening for discovering channel activators. By targeting the gating pore as a novel channel site for new opener molecules, this work provides a tool to dissect the basic biophysical mechanisms underlying gating of VGCCs. From a translational viewpoint, it offers novel therapeutic strategies for the treatment of hyperexcitability disorders, such as epilepsy or neuropathic pain.

A number of exciting issues will certainly stimulate future investigations. Knowing the adaptability and modular nature of the VSD, could the gating pore of other voltage-gated $\mathrm{Na}^{+}, \mathrm{Ca}^{2+}$ and $\mathrm{K}^{+}$ channels accommodate small ligands and be the target of novel molecules? If so, would it be possible to trap the VSD in the resting or activated conformation and thereby design new inhibitors or openers? To what extent the gating pore shares common attributes among different VGCCs and how the selectivity of the compounds could be preserved? From a fundamental perspective, it will be important to examine the impact of these new molecules on gating currents and the effects of the surrounding lipid on their pharmacological sensitivities.

\section{Polina Kornilov ${ }^{1}$, Asher Peretz ${ }^{1}$ Bernard Attali ${ }^{1}$}

${ }^{1}$ Department of Physiology \& Pharmacology, Sackler Faculty of Medicine, Tel Aviv University, Tel Aviv 69978, Israel

Correspondence: Bernard Attali

Tel: 972-3640-5116 ; Fax: 972-3640-9113

E-mail: battali@post.tau.ac.il

\section{References}

1 Ashcroft FM. Ion channels and diseases. New York: Academic Press, 1999.

2 Swartz KJ. Nature 2008; 456:891-897.

3 Vargas E, Yarov-Yarovoy V, Khalili-Araghi F, et al. J Gen Physiol 2012; 140:587-594.

4 Long SB, Tao X, Campbell EB, et al. Nature 2007; 450:376-382. 2010; 328:67-73.

6 Li P, Chen Z, Xu H, et al. Cell Res 2013; 23:1106-1118.

7 Peretz A, Pell L, Gofman Y, et al. Proc Natl Acad Sci USA 2010; 107:15637-15642.
5 Tao X, Lee A, Limapichat W, et al. Science 